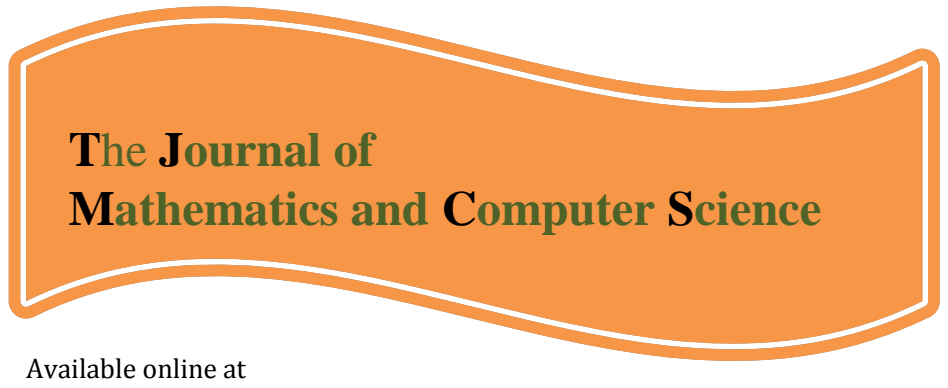

http://www.TIMCS.com

The Journal of Mathematics and Computer Science Vol .3 No.1 (2011) 1-10

\title{
EDGE EXTENSION OF GRAPHS AND DIGRAPHS
}

\author{
Akram B. Attar \\ Department of Mathematics, \\ Faculty of Education, University of Thi-Qar, Thi-Qar, Iraq \\ E-mail: akramattar70@yahoo.com
}

Received: March 2011, Revised: May 2011

Online Publication: July 2011

\begin{abstract}
In this paper, the concepts of edge (arc) extension of graphs (digraphs) and the edge (arc) extensible class of graphs (digraphs) have been introduced. The classes of regular and eulerian graphs (digraphs) which are not edge (arc) extensible classes have also been introduced.

The concept of edge (arc) extensibility number has been introduced as well as the characterization of extensibility number of regular graphs (digraphs). Also the extensibility number of eulerian graphs (digraphs) has been characterized.
\end{abstract}

Keywords: Joining graphs, Extension of graphs, Regular graphs, Reducibility, Contractibility, and Connectivity

\section{Introduction}

Kharat and Waphare [6] introduced the concept of reducibility number for posts in lattices theory. Akram [3] introduced analogous concept in graph theory, in fact, he studied the reducibility of graphs (digraphs) and the characterization of reducibility number for some classes of graphs(digraphs). Akram [2] introduced the concept of contractibility number of graphs. In Akram [1] the concept of vertex extension of graphs had been introduced. In this work, we studied the concept of edge(arc) 
extension of graphs(digraphs) and the edge(arc) extensibility number for some classes of graphs(digraphs).

A graph $G=(V(G), E(G))$ consists of two finite sets, $V(G)$, the vertex set of the graph, often denoted by just $V$, which is a nonempty set of elements called vertices, and $\mathrm{E}(\mathrm{G})$, the edge set of the graph, often denoted by just $E$, which is a possibly empty set of elements called edges, such that each edge $e$ in $E$ is assigned an unordered pair of vertices $(u, v)$ called the end vertices of $e$.The number of vertices of $G$ will be called the order of $G$, and will usually be denoted by $p$; the number of edges of $G$ will generally be denoted by $q$. If for a graph $G, p=1$ then $G$ is called trivial graph; if $q=0$ then $G$ is called a null graph. We shall usually denote the edge corresponding to $(v, w)$ where $(v$ and $w$ are vertices of $G$ ) by $v w$.

If $e$ is an edge of $G$ having end vertices $v, w$ then $e$ is said to join the vertices $v$ and $w$, and these vertices are then said to be adjacent. An independent set of vertices in $G$ is a set of vertices of $G$ no two of which are adjacent.

Let $v$ be a vertex of the graph $G$. If $v$ joined to itself by an edge, such an edge is called loop. The degree $d(v)$ is the number of edges of $G$ incident with $v$, counting each loop twice. If two (or more) edges of $G$ have the same end vertices then these edges are called parallel. A graph is called simple if it has no loops and parallel edges. We say that $G$ is r-regular graph if the degree of every vertex is $r$.

A graph $G$ is connected if there is a path joining each pair of vertices of $G$; a graph which is not connected is called disconnected.

A directed graph $D=(V, A)$ consists of two finite sets $V$, the vertex set, a nonempty set of elements called the vertices of $D$ and $A$, the arc set, a possible empty set of elements called the $\operatorname{arcs}$ of $D$, such that each arc $a$ in $A$ is assigned an ordered pair of vertices $(u, v)$.

If $a$ is an arc, in the directed graph $D$, with associated ordered pair of vertices $(u, v)$, then $a$ is said to join $u$ to $v$.

A vertex $v$ of the digraph $D$ is said to reachable from a vertex $u$ if there is a directed path in $D$ from $u$ to $v$. A digraph $D$ is said to be connected if its underlying graph is connected. A digraph $D$ is called simple if, for any pair of vertices $u$ and $v$ of $D$, there is at most one arc from $u$ to $v$ and there is no arc from $u$ to itself.

Let $v$ be a vertex in the digraph $D$. The indegree $i d(v)$ of $v$ is the number of arcs of $D$ that have $v$ as its head, i.e., the number of arcs that "go to" $v$. Similarly, the outdegree $\operatorname{od}(v)$ of $v$ is the number of arcs of $D$ that have $v$ as its tail, i.e., that "go out" of $v$. 
For the undefined concepts and terminology we refer the reader to Wilson[9], Clark[4], Harary[5], West[8] and Tutte[7].

All the graphs(digraphs) through out this paper are nontrivial and simple.

\section{Edge Extensibility of Graphs.}

In this section, we introduced the concepts of edge extension set of graph, edge extensible class of graphs and the edge extensibility number of graph. Further, we characterized the edge extensibility number of regular and eulerian graphs.

Definition 2.1: Let $G$ be a nontrivial (not complete) simple graph. The simple graph obtained from $G$ by adding a nonempty set of edges $S$ such that every edge in $S$ join two nonadjacent vertices in $G$ is called edge extension graph, and denoted by $G+S, S$ is called edge extension set. In particular, if $S$ consists of a single element $e$, then $e$ is called extension edge, and the graph denoted by $G+e$.

We can see that the graph $G+S$ have vertex set and edge set as follows:

$$
\begin{aligned}
& V(G+S)=V(G), \\
& E(G+S)=E(G) \bigcup S
\end{aligned}
$$

Definition 2.2: Let $\mathfrak{I}$ be a class of graphs satisfy certain property. Then $\mathfrak{I}$ is called edge extensible class, if for every graph $G \in \mathfrak{I}$, either $G$ is complete, or there exists an extension edge $e$ such that $G+e \in \mathfrak{I}$.

\section{Examples 2.3:}

1. The class of connected graphs is edge extensible class.

2. The class of regular graphs is not edge extensible class.

3. The class of eulerian graphs is not edge extensible class.

Definition 2.4: let $\mathfrak{I}$ be a class of graphs with certain property, and $G \in \mathfrak{I}$ be a nontrivial. The edge extensibility number of $G$ with respect to $\mathfrak{I}$ is the smallest positive integer $m$, if exists, such that there exists an edge extension set $S$ of cardinality $m$ in such away the graph $G+S \in \mathfrak{I}$. We write $m=\underset{\mathfrak{I}}{\operatorname{ext}}(G)$. If such a number does not exist for $G$, then we say that the corresponding edge extensibility number is $\infty$.

One can see that the tree $T$ with respect to the class of trees $\mathfrak{I}$ has extensibility number is $\infty$. Further, the class of graphs $\mathfrak{I}$ is edge extensible if and only if for every graph $G \in \mathfrak{I}$ either $G$ is complete or $G$ has extensibility number one. 
Now we characterize the edge extensibility number for regular graphs.

Theorem 2.5: Let $\mathfrak{R}$ be the class of regular graphs, and $R \in \mathfrak{R}$ be a nontrivial simple r-regular graph of order $P$ with $r \neq P-1$. Then

$e-\underset{\Re}{\operatorname{ext}}(R)= \begin{cases}\mathrm{P} / 2 & \text { if and only if } \mathrm{P} \text { is even } \\ P & \text { if and only if } \mathrm{P} \text { is odd }\end{cases}$

Proof: Let $R$ be an r-regular graph of order $P$ with $r \neq P-1$. Then $d(u)=r \quad \forall u \in R$. Let $S$ be a nonempty set of edges. If $R+S$ is regular, then $d(v)=r+h \forall v \in R+S$. That means the degree of every vertex in $R$ increased by $h$ where $h$ is a positive integer. It is clear that this happened when $S$ forms an n-factor in the graph $R+S$.

Suppose that $P$ is even. As $r \neq P-1$, then every vertex in $R$ is not adjacent to at least one vertex. Let $S=\left\{e_{1}, e_{2}, \ldots, e_{p / 2}\right\}$ be a set of edges and join every edge in $S$ to two nonadjacent vertices in $R$ such that $S$ forms a 1-factor in the graph $R+S$. Then $d(w)=r+1 \quad \forall w \in R+S$. Thus the graph $R+S$ is $(r+1)-$ regular and $S$ is edge extension set of cardinality $p / 2$ of $R$. Hence $e-\operatorname{ext}_{\mathfrak{R}}(R) \leq p / 2$. If $e-\operatorname{ext}_{\mathfrak{R}}(R) \neq p / 2$, then there exists an edge extension set $L=\left\{e_{1}, e_{2}, \ldots, e_{n}\right\}$ with $n<p / 2$ such that $R+L \in \mathfrak{R}$ which is impossible as $L$ can not be a 1 -factor in $R+L$ and then joining the nonadjacent vertices of $R$ by the edges of $L$ does not give a regular graph. Hence $e-\underset{\Re}{\operatorname{ext}}(R)=p / 2$.

Suppose that $P$ is odd. Let $S=\left\{e_{1}, e_{2}, \ldots, e_{P}\right\}$ be a set of edges and join every edge in $S$ to two nonadjacent vertices in $R$ such that $S$ forms a 2-factor in the graph $R+S$. Then the graph $R+S$ is $(r+2)$ - regular and $S$ is edge extension set of cardinality $P$ of $R$. Then $e-\operatorname{ext}_{\mathfrak{R}}(R) \leq P$. If $e-\operatorname{ext}_{\mathfrak{R}}(R) \neq P$, then there exists an edge extension set $F=\left\{e_{1}, e_{2}, \ldots, e_{n}\right\}$ with $n<P$ such that $R+F \in \mathfrak{R}$. Thus $F$ forms an n-factor which is impossible as $n<P$ and $P$ is odd. Hence $e-\underset{\Re}{\operatorname{ext}}(R)=P$.

Conversely, suppose that $e-\operatorname{ext}_{\Re}(R)=p / 2$, then by definition 2.4 , there exists an edge extension set $S$ of cardinality $p / 2$, and $p / 2$ is the smallest positive integer such that $R+S \in \mathfrak{R}$. As $p / 2$ is integer $P$ must be even.

Suppose that $e-\operatorname{ext}_{\mathfrak{R}}(R)=P$. By definition 2.4, there exists an edge extension set $S$ of cardinality $P$, and $S$ is the smallest set such that $R+S \in \mathfrak{R}$. If $P$ is even then there exists an edge extension set $A$ with cardinality $p / 2$ such that $R+A \in \mathfrak{R}$ and by part one $e-\underset{\Re}{\operatorname{ext}}(R)=p / 2$, which is a contradiction to our assumption. Hence $P$ is odd. 
In the following theorem, we characterize the edge extensibility number for eulerian graphs.

Theorem 2.6: Let $\mathrm{E}$ be the class of eulerian graphs, and $G \in \mathrm{E}$. Then $e-\underset{\mathrm{E}}{\operatorname{ext}}(G)=3$ if and only if $G$ contains a set of three independent vertices.

Proof: Suppose that $e-\underset{\mathrm{E}}{\operatorname{ext}}(G)=3$. Then by definition 2.4, there exists an edge extension set $S=\left\{e_{1}, e_{2}, e_{3}\right\}$ of cardinality 3 such that $G+S \in \mathrm{E}$, and $S$ is the smallest such set.

As $G$ is eulerian, then $G$ is connected and the degree of every vertex in $G$ is even. By definition 2.1, $G+S$ is simple, then the set of edges $S=\left\{e_{1}, e_{2}, e_{3}\right\}$ in the graph $G+S$ take one of the five forms $3 P_{1}, P_{1} \cup P_{2}, P_{3}, K_{1,3}$ or $C_{3}$.

As $G+S$ is eulerian, every vertex in $G+S$ has even degree, and it is clear this happened only when $S$ of the form $C_{3}$. Hence $e_{1}, e_{2}$ and $e_{3}$ are the edges of the cycle $C_{3}$. As every edge in $S$ join two nonadjacent vertices in $G$, then the vertices of $C_{3}$ are independent set of vertices in $G$.

Conversely, suppose that $A=\left\{v_{1}, v_{2}, v_{3}\right\}$ is a set of independent vertices in the eulerian graph $G$; let $S=\left\{e_{1}, e_{2}, e_{3}\right\}$ be a set of edges. Join every edge in $S$ to two nonadjacent vertices in $A$. Thus $S$ forms a cycle $C_{3}$ in the graph $G+S$. That is every vertex in $G+S$ has an even degree. As adding the edges to a connected graph preserves the connectedness, then $G+S$ is connected. Hence $G+S$ is eulerian and $S$ is edge extension set of cardinality 3 . Hence $e-\underset{\mathrm{E}}{\operatorname{ext}}(G) \leq 3$.

Suppose that $e-\underset{\mathrm{E}}{\operatorname{ext}}(G)=2$. Then there exists an edge extension set $L=\left\{e_{1}, e_{2}\right\}$ of cardinality 2 such that $G+L \in \mathrm{E}$, which is impossible, as by definition $2.1, G+L$ is simple, the set $L$ in the graph $G+L$ take one of the two forms $P_{2}$ or $2 P_{1}$, it is obvious in each case $G+L \notin \mathrm{E}$.

As $G+e$ is simple , $G+e$ is not eulerian. Hence $e-\underset{\mathrm{E}}{\operatorname{ext}}(G) \neq 1$.

Thus $e-\underset{\mathrm{E}}{\operatorname{ext}}(G)=3$. 


\section{Arc Extension Digraphs}

In this section the arc extension digraph has been introduced. Further, the arc extensibility number for regular and eulerian digraphs has been characterized. First we need to define the symmetric digraph.

Definition 3.1: Let $D$ be a digraph, the pair of vertices $u, v$ in $D$ is called symmetric if there is an arc from $u$ to $v$ and arc from $v$ to $u$. If every pair of vertices in $D$ is symmetric then $D$ is called symmetric digraph. If $D$ does not contain any symmetric pair, then $D$ is called antisymmetric.

By similar way to that in definition 2.1, we define the arc extension digraph.

Definition 3.2: Let $D$ be a not symmetric (nontrivial) simple digraph. The simple digraph obtained from $D$ by adding a nonempty set of $\operatorname{arcs} S$ such that every arc in $S$ join a pair of vertices $u, v$ in which there is no arc from $u$ to $v$ is called arc extension digraph, denoted by $D+S, S$ is called arc extension set. Inparticular, if $S$ consists of a single element $a$, then $a$ is called extension $\operatorname{arc}$, and the digraph denoted by $D+a$.

Now we define the arc extensible class of digraphs.

Definition 3.3: Let D be a class of digraphs satisfying certain property. The class D is called arc extensible if for every $D \in \mathrm{D}$ either $D$ is symmetric or there exists an extension arc $a$ such that $D+a \in \mathrm{D}$.

One can see that the class of connected digraphs is arc extensible, but neither the class of regular digraphs nor the class of eulerian digraphs is arc extensible.

The definition of arc extensibility number is similar to that in definition 2.4 , only replace each graph by a "digraph" and each edge by an "arc" as follows:

Definition 3.4: let D be a class of a digraphs with certain property, and $D \in \mathrm{D}$ be a nontrivial. The arc extensibility number of $D$ with respect to $\mathrm{D}$ is the smallest positive integer $m$, if exists, such that there exists an arc extension set $S$ of cardinality $m$ in which the digraph $D+S \in \mathrm{D}$. We write $m=a-\underset{\mathrm{D}}{\operatorname{ext}}(D)$. If such a number does not exist for $D$, then we say that the corresponding arc extensibility number is $\infty$.

Definition 3.5: Two (or more) directed cycles in the digraph $D$ are called disjoint if its arcs and vertices are distinct.

Here we characterized the arc extensibility number for the regular digraphs.

Theorem 3.6: Let $\mathrm{D}$ be a class of regular digraphs, $D \in \mathrm{D}$ be an 
antisymmetric simple regular digraph of $P$ vertices. Then $a-\underset{\mathrm{D}}{\operatorname{ex}}(D)=P$ if and only if $D$ contains a set of disjoint directed cycles $C$ such that every vertex in $D$ belongs to a cycle in $\mathrm{C}$.

Proof: Suppose that $a-\underset{\mathrm{D}}{\operatorname{ext}}(D)=P$. Then by definition 3.4, there exists an arc extension set $S=\left\{a_{1}, a_{2}, \ldots, a_{p}\right\}$ of cardinality $P$ such that $D+S \in \mathrm{D}$ and $S$ is the smallest such set.

As $D+S$ is regular digraph, then $i d(v)=o d(v)=r, \forall v \in D+S$. As the cardinality of $S$ equals to $P$ and $D+S$ is regular, then either $S$ forms a directed Hamiltonian cycle in $D+S$ or $S$ forms a set of disjoint directed cycles pass through all the vertices of $D$.

Suppose that $S$ forms the directed cycle $u_{1} a_{1} u_{2} a_{2} u_{3} \ldots u_{p} a_{p} u_{1}$ in the digraph $D+S$. By the definition $3.2, D+S$ is simple digraph. Then we must have the directed cycle $u_{1} f_{p} u_{p} f_{p-1} u_{p-1} \ldots u_{2} f_{1} u_{1}$ in $D$.

Suppose that $S$ forms a set of disjoint directed cycles pass through all the vertices of $D$. Then by similar argument for all the cycles in $S$ as above, $D$ must have a set of disjoint cycles pass through all the vertices of $D$.

Conversely, suppose that $D$ contains a set of disjoint directed cycles $C$ such that every vertex in $D$ belongs to a cycle in $\mathrm{C}$.

We discuss the proof when $C$ consists of a single element and the other cases is by similar argument.

Suppose that $\mathrm{C}$ consists of a unique directed cycle $v_{1} f_{1} v_{2} f_{2} v_{3} \ldots v_{p} f_{p} v_{1}$ in $D$. By assumption every vertex in $D$ belongs to a cycle in C. Then $v_{1} f_{1} v_{2} f_{2} v_{3} \ldots v_{p} f_{p} v_{1}$ is Hamiltonian directed cycle in $D$.

Let $H=\left\{h_{1}, h_{2}, \ldots, h_{p}\right\}$ be a set of arcs. As $D$ is antisymmetric, we can join $v_{1}$ to $v_{p}$ by $h_{p} ; v_{p}$ to $v_{p-1}$ by $h_{p-1} ; \ldots ; v_{2}$ to $v_{1}$ by $h_{1}$. Then $v_{1} h_{p} v_{p} h_{p-1} v_{p-1} \ldots v_{2} h_{1} v_{1}$ is a Hamiltonian directed cycle in the digraph $D+H$ and $i d(w)=o d(w)=r \quad \forall w \in D+H$. Thus $D+H$ is regular digraph and $H$ is arc extension set of cardinality $P$. Hence $a-\underset{\mathrm{D}}{\operatorname{ext}}(D) \leq P$. If $a-\underset{\mathrm{D}}{\operatorname{ext}}(D) \neq P$, then there exists an arc extension set $L=\left\{l_{1}, l_{2}, \ldots, l_{n}\right\}$ with $n<P$ such that $D+L \in \mathrm{D}$.

As $D+L \in \mathrm{D}$, then $i d(v)=o d(v)=r, \forall v \in D+L$. That means either $L$ forms a Hamiltonian directed cycle or $L$ forms a set of disjoint directed cycles pass through all vertices of $D$, which is impossible as $n<p$. 
Hence $a-\underset{\mathrm{D}}{\operatorname{ext}}(D)=P$.

Now, we characterize the arc extensibility number for the eulerian digraphs.

Theorem 3.7: Let $\mathrm{E}$ be the class of eulerian digraphs, $D \in E$ be a simple. Then $a-\underset{\mathrm{E}}{\operatorname{ext}}(D)=2$ if and only if $D$ contains two independent vertices.

Proof: Suppose that $a-\underset{\mathrm{E}}{\operatorname{ext}}(D)=2$, by definition 3.4, there exists an arc extension set $S=\left\{a_{1}, a_{2}\right\}$ of cardinality two such that $D+S \in E$, and $S$ is the smallest such set.

As the digraph $D+S$ is eulerian, then $D+S$ is connected and $i d(v)=o d(v), \quad \forall v \in D+S$, clearly this happen when the set of arcs $S$ forms a directed cycle. As $S$ consists of two arcs $a_{1}, a_{2}$, then $S$ forms a directed cycle of length two in $D+S$, in such away $a_{1}, a_{2}$ are joined two independent vertices in $D$.

Conversely, suppose that $u, v$ are two independent vertices in the eulerian digraph $D$, and $S=\left\{a_{1}, a_{2}\right\}$ is a set of two arcs.

Join the vertex $u$ to $v$ by the arc $a_{1}$ and $v$ to $u$ by $a_{2}$. Then $S$ forms a directed cycle of length two. Thus $i d(w)=o d(w) \forall w \in D+S$. As the addition of arcs to a connected graph preserves the connectedness, then $D+S$ is connected. Hence the digraph $D+S$ is eulerian, and $S$ is arc extension set of cardinality 2 . That is $a-\underset{\mathrm{E}}{\operatorname{ext}}(D) \leq 2$. If $a-\underset{\mathrm{E}}{\operatorname{ext}}(D) \neq 2$, then there exists an extension arc $a$ such that $D+a \in E$ which is clearly impossible. Hence $a-\underset{\mathrm{E}}{\operatorname{ext}}(D)=2$.

Theorem 3.8: Let $\mathrm{E}$ be the class of eulerian digraphs, $D \in E$ be a simple and antisymmetric. Then $a-\underset{\mathrm{E}}{\operatorname{ext}}(D)=k, \quad k>2$ if and only if $D$ does not contain two independent vertices and the smallest directed cycle in $D$ has a length $k$.

Proof: Suppose that $a-\underset{\mathrm{E}}{\operatorname{ext}}(D)=k, k>2$, by definition 3.4, there exists an arc extension set $S=\left\{a_{1}, a_{2}, \ldots, a_{k}\right\}$ of cardinality $k$ such that $D+S \in E$, and $S$ is the smallest such set.

If $D$ contains two independent vertices, then by theorem 3.7, $a-\underset{\mathrm{E}}{\operatorname{ext}}(D)=2$ a contradiction to our assumption that $k>2$.

As $D+S$ is eulerian, then $D+S$ is connected and $i d(w)=o d(w) \forall w \in D+S$. As $D$ is antisymmetric and $i d(w)=o d(w) \quad \forall w \in D+S$, then either $S$ forms a directed cycle or $S$ forms a union of directed cycles in $D+S$. 
Suppose that $S$ forms the directed cycle $u_{1} a_{1} u_{2} a_{2} \ldots u_{k} a_{k} u_{1}$ in $D+S$. As by definition 3.2, $D+S$ is simple, we must have the directed cycle $u_{1} b_{k} u_{k} b_{k-1} u_{k-1} \ldots u_{2} b_{1} u_{1}$ in $D$. Thus $D$ contains the direct cycle $C_{k}$ of length $k$.

Suppose that $D$ contains a directed cycle $C_{n}$ of length $n<k$ and denote $C_{n}$ by $v_{1} d_{1} v_{2} d_{2} \ldots v_{n} d_{n} v_{1}$, then there exists an arc extension set $H=\left\{f_{1}, f_{2}, \ldots, f_{n}\right\}$ such that $v_{1} f_{n} v_{n} f_{n-1} v_{n-1} \ldots v_{2} f_{1} v_{1}$ forms a cycle in $D+S$, that is $i d(v)=o d(v) \forall v \in D+H$, and as the adding of arcs to a connected graph preserves the connectedness, $D+S$ is eulerian and $a-\underset{\mathrm{E}}{\operatorname{ext}}(D)=n$ which is a contradiction to our assumption that $a-\underset{\mathrm{E}}{\operatorname{ext}}(D)=k$. Hence $C_{k}$ is the smallest directed cycle in $D$.

Suppose that $S$ forms a union of cycles in $D+S$, then by consider each of these cycles and using the same argument above, we get a contradiction to the minimality of $k$.

Conversely, suppose that $D$ does not contain two independent vertices and the smallest directed cycle in $D$ has a length $k$.

Let $u_{1} b_{1} u_{2} b_{2} u_{3} \ldots u_{k} b_{k} u_{1}$ be a cycle of length $k$ in $D$; let $S=\left\{a_{1}, a_{2}, \ldots, a_{k}\right\}$ be a set of arcs. Join $u_{1}$ to $u_{k}$ by $a_{k} ; u_{k}$ to $u_{k-1}$ by $a_{k-1} ; \ldots ; u_{2}$ to $u_{1}$ by $a_{1}$. Then $u_{1} a_{k} u_{k} a_{k-1} u_{k-1} \ldots u_{2} a_{1} u_{1}$ forms a directed cycle in $D+S$. Then the digraph $D+S$ is connected and $i d(w)=o d(w) \quad \forall w \in D+S$. Hence $D+S$ is eulerian and $S$ is an arc extension set of cardinality $k$. Thus $a-\underset{\mathrm{E}}{\operatorname{ext}}(D) \leq k$. If $a-\underset{\mathrm{E}}{\operatorname{ext}}(D) \neq k$, then there exists an arc extension set $H=\left\{f_{1}, f_{2}, \ldots, f_{n}\right\}$ with $n<k$ such that $D+H \in E$. By part one $H$ must be forms a directed cycle in $D+H$, say $v_{1} f_{1} v_{2} f_{2} v_{3} \ldots v_{n} f_{n} v_{1}$. In this case $v_{1} h_{n} v_{n} h_{n-1} v_{n-1} \ldots v_{2} h_{1} v_{1}$ is a directed cycle in $D$. Hence $D$ contains a cycle of length $n<k$ a contradiction to the smallestness of $k$. Hence $a-\underset{\mathrm{E}}{\operatorname{ext}}(D)=k$. As $D$ does not contain two independent vertices, then $k>2$.

\section{REFERENCES}

[1] Akram B. Attar "Extensibility of Graphs". Journal of Applied Mathematics, Islamic Azad University of Lahijan Vol.6, No.21, Summer[ 2009].

[2] Akram B. Attar: Contractibility of Regular Graphs. J. C. E Al-Mustanseriya Uni. No.3 [2007].

[3] Akram B. Attar: Reducibility For Certain Classes of Graphs and Digraphs. Ph. D thesis Pune Uni. India [2005]. 
Akram B. Attar / TJMCS Vol .3 No.1 (2011) 1-10

[4] Clark, J. and Holton, D.A: A First Look at Graph Theory, World Scientific, London.[1991].

[5] Harary, F.: Graph Theory, Addison-Wesley, Reading MA [1969].

[6] Kharat, V. S. and Waphare, B. N.: Reducibility in finite posets, Europ. J. Combinatorics bf 22 [2001] , 197-205.

[7] Tutte, W. T: Graph Theory, Addison-Wesley [1984].

[8] West, D. B. : Introduction to Graph Theory, University of Illinois-Urbana, Prentice Hall of India [1999].

[9] Wilson, R. J. and Beineke, L. W.: Selected Topics in Graph Theory, Academic Press, London [1978]. 